Marquette University

e-Publications@Marquette

Biomedical Sciences Faculty Research and

Publications

Biomedical Sciences, Department of

4-2017

\title{
The Application of CRISPR Technology to High Content Screening in Primary Neurons
}

\author{
Ben L. Callif \\ Marquette University \\ Brian Maunze \\ Marquette University, brian.maunze@marquette.edu \\ Nick L. Krueger \\ Marquette University \\ Matthew T. Simpson \\ Marquette University \\ Murray G. Blackmore \\ Marquette University, murray.blackmore@marquette.edu
}

Follow this and additional works at: https://epublications.marquette.edu/biomedsci_fac

Part of the Neurosciences Commons

\section{Recommended Citation}

Callif, Ben L.; Maunze, Brian; Krueger, Nick L.; Simpson, Matthew T.; and Blackmore, Murray G., "The Application of CRISPR Technology to High Content Screening in Primary Neurons" (2017). Biomedical Sciences Faculty Research and Publications. 184.

https://epublications.marquette.edu/biomedsci_fac/184 
Marquette University

\section{e-Publications@Marquette}

\section{Biomedical Sciences Faculty Research and Publications/College of Health Sciences}

This paper is NOT THE PUBLISHED VERSION; but the author's final, peer-reviewed manuscript. The published version may be accessed by following the link in the citation below.

Molecular and Cellular Neuroscience, Vol. 80 (2017): 170-179. DOI. This article is (C) Elsevier and permission has been granted for this version to appear in e-Publications@Marquette. Elsevier does not grant permission for this article to be further copied/distributed or hosted elsewhere without the express permission from Elsevier.

\section{The Application of CRISPR Technology to High Content Screening in Primary Neurons}

Ben L. Callif

Marquette University, Milwaukee WI

Brian Maunze

Marquette University, Milwaukee WI

Nick L. Krueger

Marquette University, Milwaukee WI

Matthew T. Simpson

Marquette University, Milwaukee WI

Murray G. Blackmore

Marquette University, Milwaukee WI 


\section{Abstract}

Axon growth is coordinated by multiple interacting proteins that remain incompletely characterized.

High content screening (HCS), in which manipulation of candidate genes is combined with rapid image analysis of phenotypic effects, has emerged as a powerful technique to identify key regulators of axon outgrowth. Here we explore the utility of a genome editing approach referred to as CRISPR (Clustered Regularly Interspersed Palindromic Repeats) for knockout screening in primary neurons. In the CRISPR approach a DNA-cleaving Cas enzyme is guided to genomic target sequences by user-created guide RNA (sgRNA), where it initiates a double-stranded break that ultimately results in frameshift mutation and loss of protein production. Using electroporation of plasmid DNA that co-expresses Cas9 enzyme and sgRNA, we first verified the ability of CRISPR targeting to achieve protein-level knockdown in cultured postnatal cortical neurons. Targeted proteins included NeuN (RbFox3) and PTEN, a wellstudied regulator of axon growth. Effective knockdown lagged at least four days behind transfection, but targeted proteins were eventually undetectable by immunohistochemistry in $>80 \%$ of transfected cells. Consistent with this, anti-PTEN sgRNA produced no changes in neurite outgrowth when assessed three days post-transfection. When week-long cultures were replated, however, PTEN knockdown consistently increased neurite lengths. These CRISPR-mediated PTEN effects were achieved using multi-well transfection and automated phenotypic analysis, indicating the suitability of PTEN as a positive control for future CRISPR-based screening efforts. Combined, these data establish an example of CRISPR-mediated protein knockdown in primary cortical neurons and its compatibility with HCS workflows.

\section{Graphical abstract}

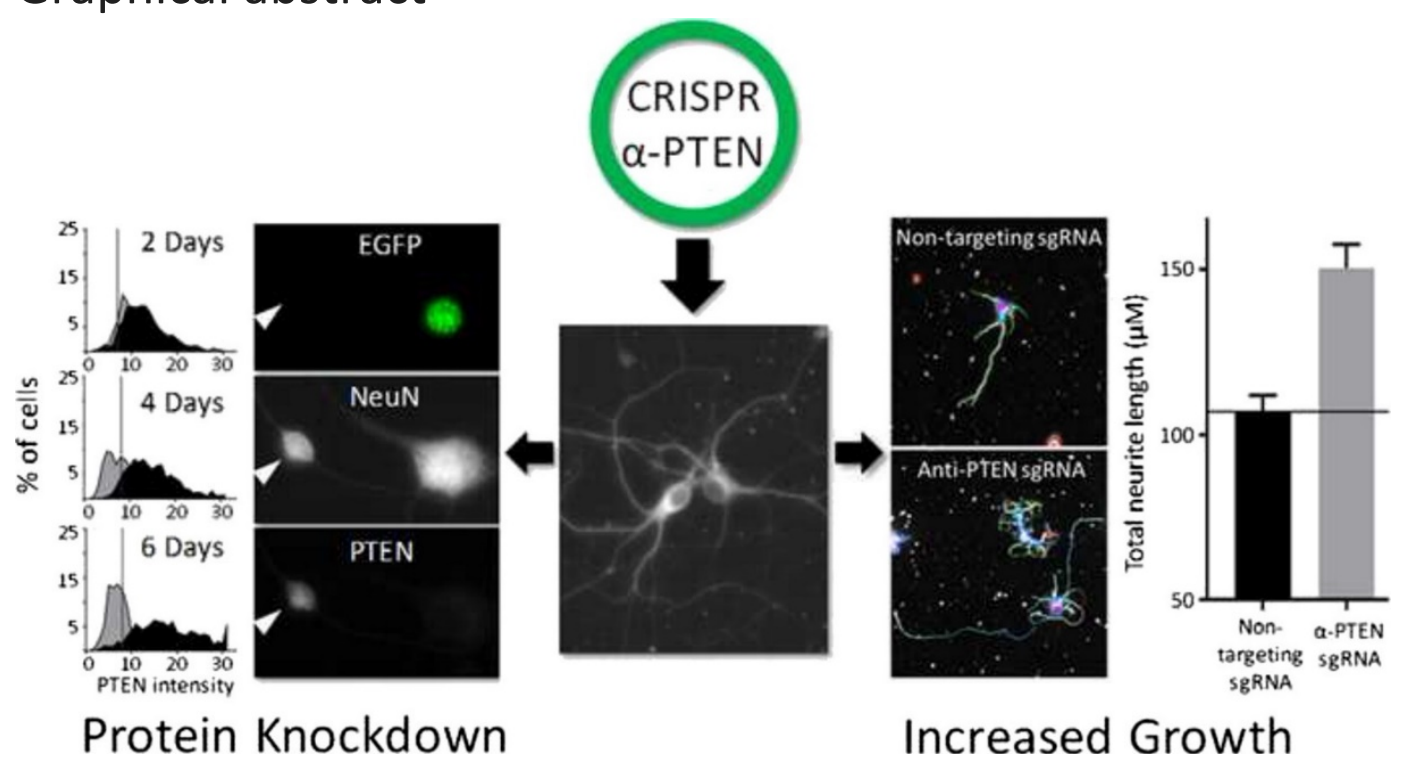

\section{Keywords}

High content screening, CRISPR, Cortical neuron, PTEN

\section{Introduction}

The growth of axons and dendrites depends on numerous cellular processes including cytoskeletal remodeling, membrane expansion, intracellular signaling, regulated transport, and transcription. Thus, 
the coordinated activity of hundreds of proteins is needed for effective neurite extension. Identifying the full set of proteins that regulate axon growth remains a key objective in regenerative neuroscience and is critical for ongoing efforts to improve regenerative ability in neurons that have lost axons to injury or disease. For instance, it is now clear that targeted manipulation of gene expression in injured neurons is an effective means to improve axon growth (Belin et al., 2015, Blackmore et al., 2012, Sun et al., 2011, Wang et al., 2015). Improvements remain partial, however, motivating the search for additional regulatory proteins that might act as therapeutic targets.

Forward genetic screens performed in primary neurons have emerged as a powerful tool to discover novel regulators of neurite growth (Blackmore, 2012). These screens generally rely on two components. First, approaches such as electroporation or viral transduction in multi-well format are used to achieve high throughput genetic modification of primary neurons. Second, automated image analysis in multi-well format rapidly quantifies effects on neurite extension. This readout can be performed on the overall population of neurons (e.g. "well-based" quantification), or can assess neurite length from individual neurons (e.g. "cell-based" analysis). A variety of labs have now harnessed this genetic screening approach, leading to a rapid expansion of the set of known regulators of axon growth (Blackmore, 2012, Blackmore et al., 2010, Buchser et al., 2012, Simpson et al., 2015, Zou et al., 2015). Many of these screens involve gene overexpression and thus address the sufficiency of ectopic gene expression to evoke phenotypic responses. To clarify the role of endogenous protein in neurite outgrowth, however, knockout approaches are necessary (e.g. Zou et al., 2015).

Genome editing methods such as zinc finger nucleases (ZFNs), Transcription Activator-Like Effector Nucleases (TALENs), and Clustered Regularly Inter-Spaced Palindromic Repeats (CRISPR) represent potentially improved tools for knockout screening. Unlike previous RNAi approaches, which act posttranscriptionally and may induce only partial protein knockdown, genome editing can modify DNA at specific loci to abolish protein production completely and permanently. CRISPR in particular has been widely adopted. CRISPR evolved as a bacterial adaptive immune system in which Cas (CRISPRassociated) nucleases are directed to degrade foreign DNA (Kunin et al., 2007). DNA binding by Cas nucleases is limited to sequences adjacent to a specific 3-nucleotide protospacer adjacent motif (PAM) (Terns and Terns, 2011). Critically, Cas nucleases are guided to specific PAM-adjacent regions by RNAs that are complementary to the target DNA sequence. It is now well established that Cas proteins are functional when expressed in mammalian cells and can be directed to specific loci by supplying RNA sequences called single-guide (sgRNA) that contain a user-designed targeting domain. Cas nucleases initiate double-stranded breaks followed by error prone non-homologous end-joining (NHEJ), resulting in insertion or deletion mutations that disrupt the production of functional protein when directed to coding regions. Importantly, examples now exist to demonstrate the efficacy of protein-level knockout by the CRISPR strategy in neurons (Platt et al., 2014, Straub et al., 2014, Swiech et al., 2015, Zuckermann et al., 2015).

There may be unique challenges, however, to adopting the CRISPR approach to screening in postmitotic neurons. First, previous CRISPR-based screens in non-neuronal cells have relied on purifying and expanding affected cells with antibiotic resistance genes or fluorescent reporters to obtain knockout clones (An et al., 2014, Chen et al., 2015, Schmid-Burgk et al., 2016, Wang et al., 2014). Without the option of proliferation-based purification, however, gene delivery methods for neurons must be rapid and efficient. Second, no studies have yet determined how quickly CRISPR causes knockdown in neurons. There must be a defined timecourse of knockdown in order to rationally plan experimental endpoints. Finally, the few publications that have utilized CRISPR in cultured neurons have used lentiviral-based approaches to force expression of a Cas enzyme and an sgRNA (Incontro et 
al., 2014, Telese et al., 2015). Large-scale screening, however, may be more feasible and economical using plasmid-based transfection (Ebert et al., 2008, Moffat et al., 2006, Root et al., 2006).

Here we performed validation and optimization experiments to assess the utility of CRISPR for high content screening in neurons. Using delivery of Cas9 and sgRNA by plasmid electroporation in multiwell format, we first confirmed the timecourse of protein knockdown using a previously validated targeting sequence for NeuN (Rbfox3), a ubiquitously expressed neuronal protein (Platt et al., 2014). We next designed and delivered sgRNA against Phosphatase and Tensin homolog (PTEN), a wellstudied inhibitor of neurite outgrowth. For both targets the majority of neurons were unaffected by CRISPR knockdown until 4 to 6 days post-transfection and during this time they extended lengthy processes that were not amenable to neurite outgrowth analysis. Therefore, we developed a replating approach to disentangle overgrown neurites. Following replating automated microscopy was able to detect a robust outgrowth phenotype from PTEN knockout, thereby establishing a positive control for CRISPR-based phenotypic screening.

\section{Methods}

\subsection{Cloning and plasmid preparation}

pX330-U6-Chimeric_BB-CBh-hSpCas9 was a gift from Feng Zhang (Addgene plasmid \# 42230). The targeting sequence for NeuN was taken from Platt et al. (2014). Targeting sequences for PTEN were designed using the publicly available Benchling CRISPR design tool (http://www.benchling.com). The overhang ' $C A C C$ ' was added to the 5 ' end of the two sgRNA sequences with the highest on-target score and the overhang 'CAAA' was added to the 5 ' end of the reverse complements of these sequences. Anti PTEN sequences were GGTGGGTTATGGTCTTCAAA (PTEN A), TTTCCTGCAGAAAGACTTGA (PTEN B), CACCGGTTTGATAAGTTCTAGCTG (PTEN C), and CACCGTTTGTGGTCTGCCAGCTAA (PTEN D). The antiNeuN sequence was CAGCAGCCCAAACGACTACAT, and the non-targeting control sequence was GGGTCTTCGAGAAGACCTGT. Oligonucleotides of the targeting sequences and reverse complements with overhangs were ordered from ThermoFisher and annealed. Bbs1 enzyme was used to digest the PX330 plasmid and the annealed oligonucleotides were ligated into the cut backbone using T4 ligase as described in Cong et al. (2013). The PX330 plasmid containing sgRNA targeting sequences were then transformed into E. cloni Chemically Competent Cells (VWR 95040-456) and DNA was prepared by QIAprep Spin Miniprep Kit (Qiagen 27,106). The reporter plasmid expressed EBFP-2A-H2B-EGFP from a CMV promoter, and was constructed by replacing the $2 \mathrm{~A}-\mathrm{mCherry}$ sequence from a reporter previously described in Wang et al. (2015) with EGFP fused to H2B to drive nuclear localization.

\subsection{Cortical cell dissociation, transfection, and culture}

All animal procedures were approved by the Marquette University Institutional Animal Care and Use Committee. Cortical neurons were prepared from early postnatal (P3-P5) Sprague Dawley rat pups (Charles River) and transfected as in Blackmore et al. (2010). Frontal cortices were placed in ice-cold Hibernate $\mathrm{E}$ (Life Technologies A12476-01), their meninges removed, finely minced and transferred into $10 \mathrm{ml}$ dissociation media for $30 \mathrm{~min}$ at $37^{\circ} \mathrm{C}$ with constant shaking (Hibernate $\mathrm{E}$ containing $20 \mathrm{U} / \mathrm{ml}$ papain (Worthington 3126) and $2.5 \mu \mathrm{g} / \mathrm{ml}$ DNAse (Sigma D4527)). Cells were then rinsed with Hibernate $E+2 \% S M 1$, then rinsed in Hibernate $E$ (no SM1), and incubated 30 min at $37{ }^{\circ} \mathrm{C}$ with constant shaking in $10 \mathrm{ml}$ trypsin solution (Hibernate E containing $0.25 \%$ trypsin (Invitrogen/Gibco 15,090-046) and $2.5 \mu \mathrm{g} / \mathrm{ml}$ DNAse (Sigma D4527)). Cells were centrifuged (1 min, $10 \mathrm{~g}$ ), washed with Hibernate $E+2 \%$ SM1, and triturated three times in $1.5 \mathrm{ml}$ Hibernate $E+2 \%$ SM1 containing $2 \mu \mathrm{l}$ 
DNAse (Sigma D4527). The suspension was allowed to settle for 2 min and the supernatant was collected; these steps were repeated until no cell pellet was visible. This process typically yielded 2 million cells $/ \mathrm{ml}$ in 6-7 $\mathrm{ml}$ Hibernate E. For transfection, cortical neurons were centrifuged (4 min, $80 \mathrm{~g}$ ) and resuspended to $2 \times 10^{6}$ cells/ml in Internal Neuronal Buffer (INB) (KCl $135 \mathrm{mM}, \mathrm{CaCl} 2$ $0.2 \mathrm{mM}, \mathrm{MgCl} 22 \mathrm{mM}$, HEPES $10 \mathrm{mM}$, EGTA $5 \mathrm{mM}$, pH 7.3). $25 \mu$ l were placed into wells of a 96-well electroporation plate (BTX Harvard Apparatus 45-0450) and mixed with $25 \mu$ of INB containing $1 \mu \mathrm{g}$ EGFP plasmid and $4 \mu \mathrm{g}$ test plasmid. A $350 \mathrm{~V}, 300 \mu$ s pulse was delivered to each well by an ECM square wave pulse generator (Harvard Apparatus ECM-830) attached to a plate handler (BTX Harvard Apparatus HT-200). After electroporation each well received $100 \mu$ of Hibernate E. For short-term cell culture experiments, 10,000 cells/well, or for long-term cell culture experiments 100,000 cells/well, were placed in 24-well plates (Greiner Bio-One 662,160) pre-coated with $100 \mu \mathrm{g} / \mathrm{ml}$ poly-d-lysine hydrobromide (Sigma P7886) (N12 h, $37^{\circ} \mathrm{C}$ ), followed by $10 \mu \mathrm{g} / \mathrm{ml} \mathrm{laminin} \mathrm{(Sigma} \mathrm{L2020)} \mathrm{(N2} \mathrm{h,} 37^{\circ} \mathrm{C}$ ). Cells were maintained at $37^{\circ} \mathrm{C}, 5 \% \mathrm{CO} 2$ in Enriched Neurobasal (ENB) media, modified from MeyerFranke et al. (1995), consisting of Neurobasal A (Invitrogen 10,888-022), 50X NeuronCult SM1 Neuronal Supplement (Stemcell Technologies 5711), 1 mM sodium pyruvate (Invitrogen 11,360-070), 2 mM GlutaMAX (Invitrogen/Gibco 35,050-061), $50 \mathrm{U} / \mathrm{ml}$ penicillin-streptomycin (Invitrogen 15,070063), $5 \mu \mathrm{M}$ forskolin (Sigma F6886),

To enrich for neurons in cultures maintained for longer than 3 days, 100 nM 5-fluoro-2'-deoxyuridine (FuDR) (Sigma-Aldrich F0503) was added to each well 1 day post-transfection. To facilitate long-term survival the media was replaced every 3 days, with fresh cortical media in the replating experiments and with FuDR-containing media in the time course experiments.

\subsection{Cortical cell culture replating}

Primary cortical neurons were transfected and cultured as previously described (Blackmore et al., 2010). Wells were rinsed twice with $400 \mu \mathrm{l}$ of Hibernate $\mathrm{E}$ and then incubated for $10 \mathrm{~min}$ at $37^{\circ} \mathrm{C}$ in $400 \mu \mathrm{l}$ of StemPro Accutase Cell Dissociation Reagent (ThermoFisher A1110501). Accutase was then diluted with $400 \mu \mathrm{l}$ of fresh cortical media, cells were detached by pipetting three times, with half the well volume aspirated and dispensed each time. Dissociated cells collected by centrifugation ( $4 \mathrm{~min}$, $80 \mathrm{G}$ ). Cells were resuspended in $1 \mathrm{ml}$, counted, and plated at 10,000/well in 24-well plates (Greiner Bio-One 662,160) pre-coated with PDL/laminin using conditioned media from the original plate. Replated cells were fixed after $24 \mathrm{~h}$ in $4 \%$ PFA.

\subsection{Cortical cell culture immunohistochemistry}

Cultures were fixed in 4\% paraformaldehyde (Electron Microscopy Sciences 15,710) for 30 min at room temperature, rinsed five times, blocked and permeabilized (PBS, 20\% goat serum (Invitrogen 16,210064), 0.2\% Triton X-100 (G-Biosciences 786-513) for $30 \mathrm{~min}$ at room temperature, and incubated in primary antibody solution (PBS, $10 \%$ goat serum, $0.2 \%$ Triton X-100, primary antibodies: rabbit antiBllltubulin (1:500, Sigma T2200), mouse anti-NeuN (1:500, Millipore MAB377), rabbit anti-PTEN (1:500, Cell Signaling 138G6) (overnight, $4{ }^{\circ} \mathrm{C}$ ). After five washes, cultures were incubated in secondary antibody solution containing DAPI nuclear stain (PBS, 10\% goat serum, $0.2 \%$ Triton X-100, $300 \mathrm{nM}$ DAPI, dilactate (molecular probes D3571) secondary antibodies: Alexa Fluor 647 goat anti-rabbit IgG (1:500, Invitrogen A-21245), Alexa Fluor 546 goat anti-mouse IgG (1:500, Invitrogen A-11003)) for $2 \mathrm{~h}$ at room temperature. Plates were washed five times, and left in $1 \mathrm{ml}$ PBS for imaging. 


\subsection{Quantification of immunofluorescence}

For the time course of PTEN knockdown experiments, a fluorescent inverted microscope was used to identify EGFP + NeuN + cells as transfected neurons. For the time course of NeuN knockdown experiments, a fluorescent inverted microscope was used to identify EGFP + BIII-tubulin + cells as transfected neurons. A circle was drawn around the cell body of each transfected cell and the fluorescence level of either NeuN or PTEN staining was recorded as quantified by NIS-Elements Basic Research software. For all treatments and time points $n=40$ transfected cells. Statistical analysis was paired $t$-test using Graphpad Prism Software.

\subsection{Neurite outgrowth analysis}

For neurite outgrowth assays, Cellomics Cell Insight NXT (Thermo Scientific) acquired images of 3 channels: nuclei (DAPI), neurite staining ( $\beta$ III-tubulin), and reporter (EGFP), and Cellomics Scan v6.4.0 (Thermo Scientific) traced neurites and quantified reporter intensity. Average total neurite length was quantified for the $10 \%$ of cells with greatest EGFP intensity per plate ( $n>100$ cells per treatment). Average neurite length was normalized to PX330 non-target control for each plate. Each treatment was tested at least three times. Statistical analysis was paired $t$-test using Graphpad Prism Software.

\section{Results}

\subsection{Time course of CRISPR-mediated knockout of target proteins in primary cortical neurons}

CRISPR has been extensively used to decrease protein expression in mitotically active cells and recently, knockdown in post-mitotic neurons has been achieved. For example, an sgRNA targeting sequence against neuron-specific NeuN was shown to reduce NeuN protein expression in adult cortical neurons in vivo upon transgenic co-expression of spCas9 (Platt et al., 2014). We therefore asked whether this same targeting sequence affected NeuN expression in primary cortical neurons that were transfected to express spCas9 and, if so, over what time course. The validated anti-NeuN targeting sequence was cloned into the Px330 plasmid, which co-expresses spCas9 and sgRNA (Cong et al., 2013). Using previously established techniques, plasmid was then delivered by electroporation to cortical neurons prepared from postnatal (P3-P5) rat pups (Blackmore et al., 2010, Buchser et al., 2006). To identify transfected cells, an EGFP reporter was co-transfected at a 1:4 ratio, a strategy shown previously to result in $>90 \%$ co-expression of test plasmid and reporter (Blackmore et al., 2010). Neurons were plated at high density $(100,000$ cells plated per well in 24 -well format) in media containing 5-Fluoro-2'-deoxyuridine (FuDR) to limit glial proliferation, and then fixed every two days between 4 and 14 days in vitro (DIV). Then, NeuN immunofluorescence was quantified in transfected neurons, which were identified by EGFP and immunohistochemistry for neuron-specific BIII-tubulin (Fig. $1 \mathrm{~A}-\mathrm{C}$ ). After 4 days, a significant but modest decrease in average NeuN immunofluorescence was detected in anti-NeuN treated cells compared to control cells transfected with SpCas9 and nontargeted sgRNA (Fig. 1D). Importantly, only $20 \%$ of anti-NeuN treated cells showed NeuN levels that fell below a threshold set by the dimmest $5 \%$ of control cells. Two days later, however, anti-NeuN displayed a larger reduction in average NeuN immunofluorescence, and $>50 \%$ of transfected cells were dimmer than the lowest $5 \%$ of control cells. Later time points showed a trend toward lower NeuN expression, with $>75 \%$ of transfected cells dimmer than $95 \%$ of control cells (Fig. 1D). These data confirm that CRISPR-mediated knockout of NeuN is effective in cultures of primary cortical neurons, but requires approximately a week to strongly affect a majority of transfected neurons. 

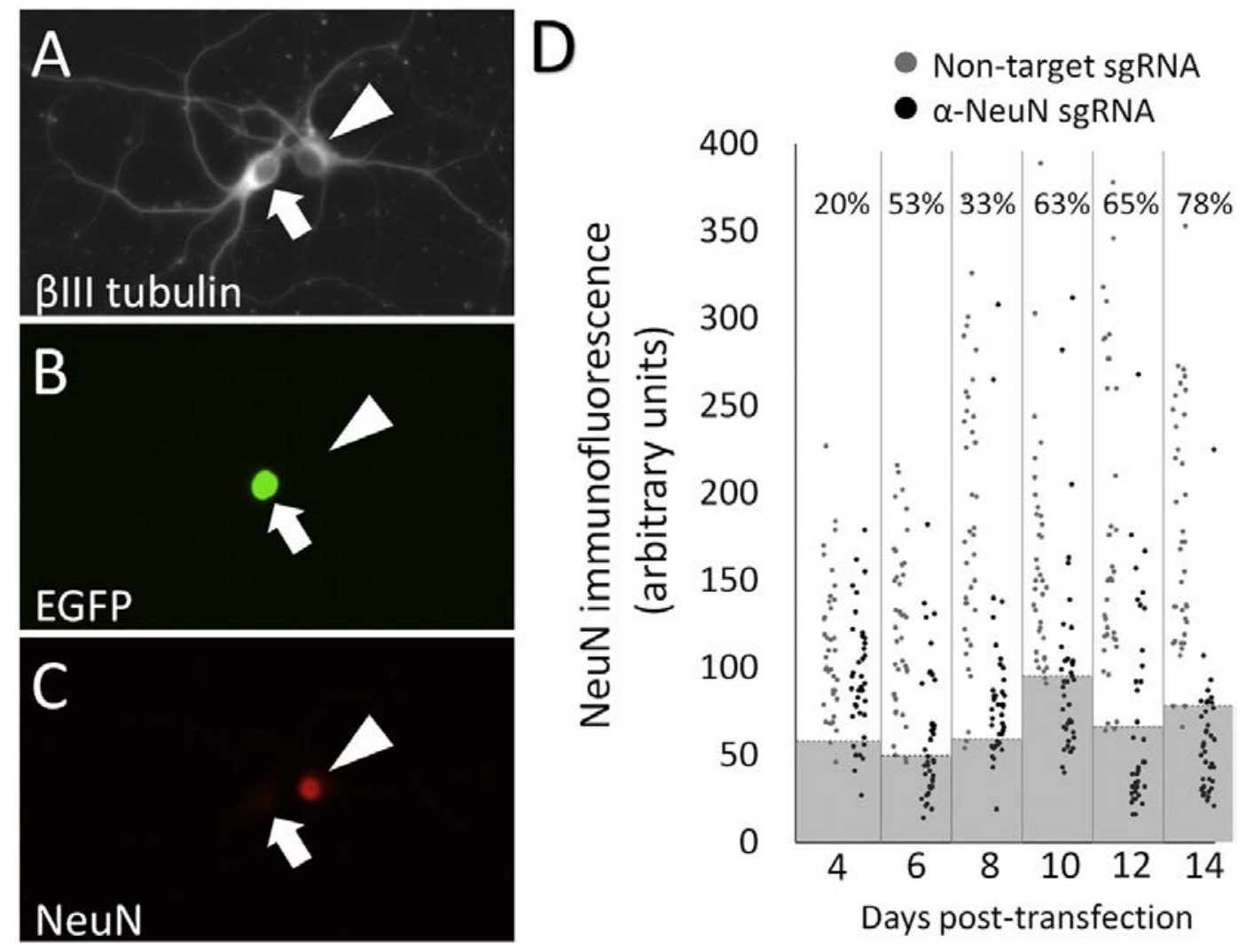

Fig. 1. CRISPR effectively decreases NeuN protein levels in primary neurons. P3-P5 cortical neurons were transfected with plasmid DNA co-expressing spCas9 and anti-NeuN or non-targeted sgRNA. Neurons were cultured, fixed between 4 and 14 days in vitro (DIV), and immunostained for neuron-specific $\beta$ III tubulin and NeuN. (A-C) show anti-NeuN treated neurons 14 days after transfection. EGFP (B) identifies transfection (arrow). (C) Immunohistochemistry detects NeuN protein in a EGFP-negative neuron (arrowhead), but not in a transfected neighbor (arrow). (D) Each dot represents NeuN immunofluorescence intensity in a single transfected (EGFP +) neuron between 4DIV and 14DIV. Grey boxes indicate a threshold set by the dimmest 5\% of control-transfected neurons at each timepoint, and the percent of anti-NeuN cells that fall below that threshold is quantified above. Significant differences existed between the population-level average intensity at all timepoints ( $p=0.033$ 4DIV, $p=7.4 \times 10^{-9} 6 \mathrm{DIV}, p=1.8 \times 10^{-8} 8 \mathrm{DIV}, p=3.7 \times 10^{-5}$ 10DIV, $p=1.6 \times 10^{-7} 12 \mathrm{DIV}$, $p=7.9 \times 10^{-12} 14 \mathrm{DIV}$; unpaired $t$-test), and the percent of anti-NeuN cells below threshold increases between 4 and 6DIV. $\mathrm{N}=40$ cells per group per timepoint.

We next sought to determine the efficacy and time course of CRISPR-based knockout of a second protein, which had not previously been targeted with a CRISPR approach. We selected PTEN for knockdown based on its well-established role in neurite outgrowth in other types of neurons (reviewed in Ohtake et al., 2015). Using publicly available algorithms to maximize selectivity and efficacy (Doench et al., 2014, Hsu et al., 2013), short oligonucleotides containing anti-PTEN targeting sequences were synthesized, annealed, and ligated into the PX330 plasmid (Cong et al., 2013). As before, P3-P5 rat cortical neurons were electroporated with $4 \mu \mathrm{g}$ of anti-PTEN or control PX330 plasmid and plated in FuDR-treated media, then fixed every 2 days starting at 4DIV and ending at 14DIV. Neurons were identified by immunohistochemistry for NeuN and transfected cells were identified by the expression of EGFP (Fig. 2A-C). PTEN expression was manually quantified by drawing a region of interest around the cell body of transfected neurons and recording average PTEN immunofluorescence, as measured by NIS-elements software. Four days after transfection, average PTEN fluorescence was significantly 
reduced in the anti-PTEN group, and $67 \%$ of transfected neurons fell below the dimmest $5 \%$ of control neurons. Two days later, average PTEN fluorescence was further reduced in the anti-PTEN group, and $85 \%$ of neurons fell below the $5 \%$ threshold (Fig. 2D). Thus, user-designed CRISPR targeting sequences effectively decreases protein expression of PTEN in primary cortical neuron cultures on a time scale of four to six days.

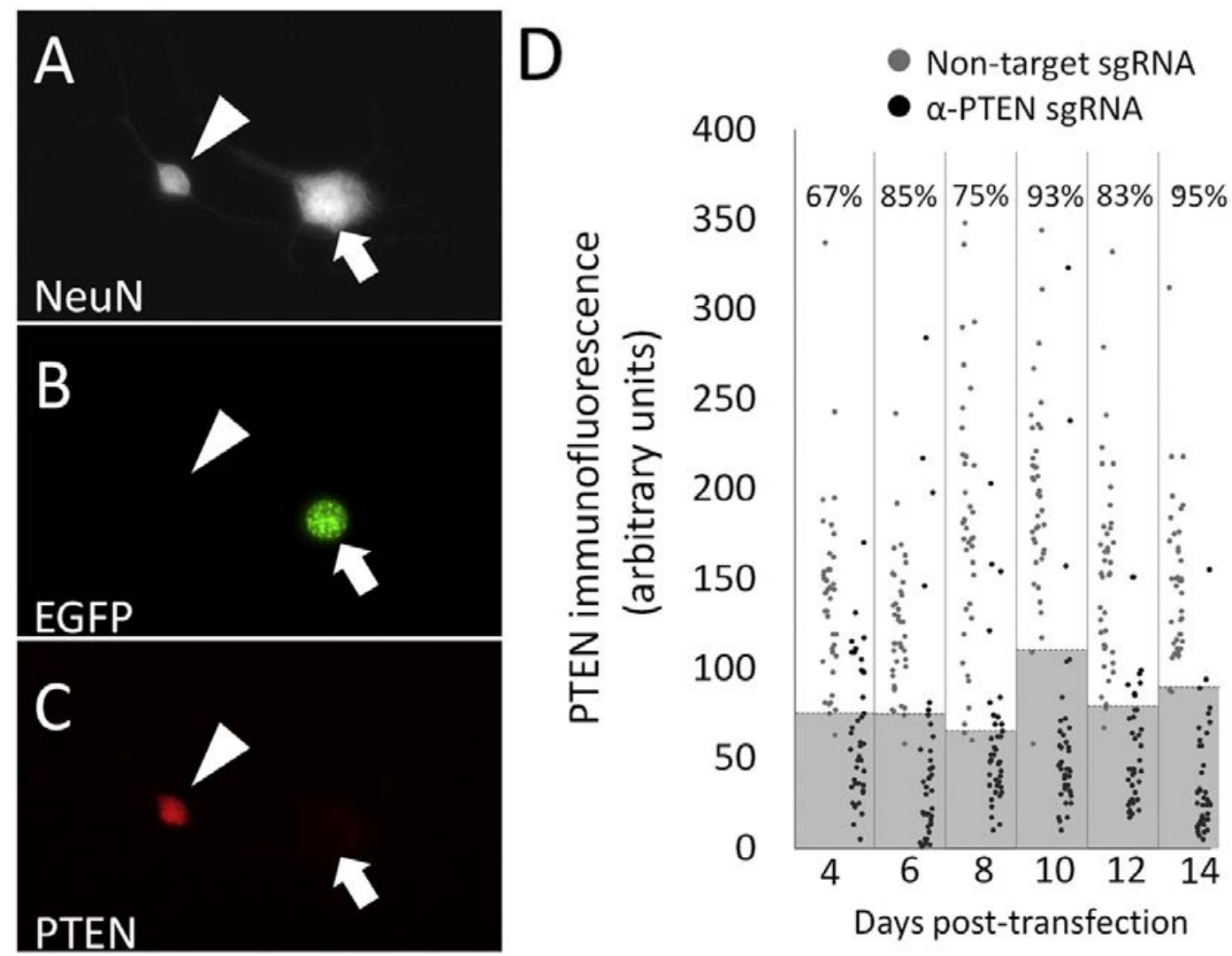

Fig. 2. CRISPR effectively decreases PTEN protein levels in primary neurons. P3-P5 cortical neurons were transfected with plasmid DNA co-expressing spCas9 and anti-PTEN or non-targeted sgRNA. (A-C) show neurons, identified by NeuN (A), 14 days after transfection with anti-PTEN. (B) EGFP identifies transfection (arrow). (C) Immunohistochemistry detects PTEN protein in a EGFP-negative neuron (arrowhead), but not in a transfected neighbor. (D) Each dot represents PTEN immunofluorescence intensity in a single transfected (EGFP +) neuron between 4DIV and 14DIV. Grey boxes indicate a threshold set by the dimmest $5 \%$ of control-transfected neurons at each timepoint, and the percent of anti-PTEN cells that fall below that threshold is quantified above.

Significant differences existed between the population-level average intensity at all timepoints $\left(p=6.9 \times 10^{-11}\right.$ 4 DIV, $p=1.4 \times 10^{-8} 6 \mathrm{DIV}, \mathrm{p}=5.0 \times 10^{-13} 8 \mathrm{DIV}, \mathrm{p}=3.3 \times 10^{-15} 10 \mathrm{DIV}, \mathrm{p}=4.8 \times 10^{-14} 12 \mathrm{DIV}, \mathrm{p}=3.7 \times 10^{-17} 14 \mathrm{DIV}$; unpaired $t$-test), and by 6 DIV $85 \%$ of anti-PTEN treated cells fell below the $5 \%$ threshold. $N=40$ cells per group per timepoint. 
One potential strategy to accelerate protein knockdown may be to use multiple sgRNAs that target discrete coding regions of the same gene. To test this possibility, four sgRNA sequences against PTEN were designed and delivered singly or in pair-wise combinations to early postnatal cortical neurons. In order to facilitate comparison with established shRNA-based approaches, cells were transfected with anti-PTEN shRNA shown previously to be effective in CNS neurons (Yungher et al., 2015). Cells were maintained between 2 and 14 days in culture, fixed, and visualized with immunohistochemistry against PTEN and neuron-specific $\beta I I I$ tubulin. In contrast to the manual quantification performed above, in this experiment we utilized a Cellomics high content screening microscope to assess PTEN intensity, which enabled analysis of hundreds of cells per treatment at each timepoint. In this analysis, the $10 \%$ of cells with the brightest EGFP signal were quantified, in order to focus the analysis on effectively transfected neurons. In support of this, we found that reduction in PTEN expression was greatest in the top $10 \%$ of EGFP-expressing cells (Supplementary Fig. 1). As shown in Fig. 3, all four anti-PTEN sgRNA sequences produced significant knockdown of PTEN in transfected neurons. Consistent with the previous manual quantification, the percent of neurons with PTEN intensity below the $5 \%$ control threshold increased from 8 to $10 \%$ at 2DIV to 51-66\% at 14DIV. Mixtures of two sgRNAs did not accelerate the onset of knockdown or increase the percent of affected cells at later timepoints. Interestingly, knockdown of PTEN protein via shRNA was much more rapid, with $58 \%$ of cells below the $5 \%$ threshold after only 2DIV. Overall, these data confirm a lag in the onset of protein knockdown by Cas9 compared to shRNA. Moreover, combined sgRNA sequences did not shorten this lag.

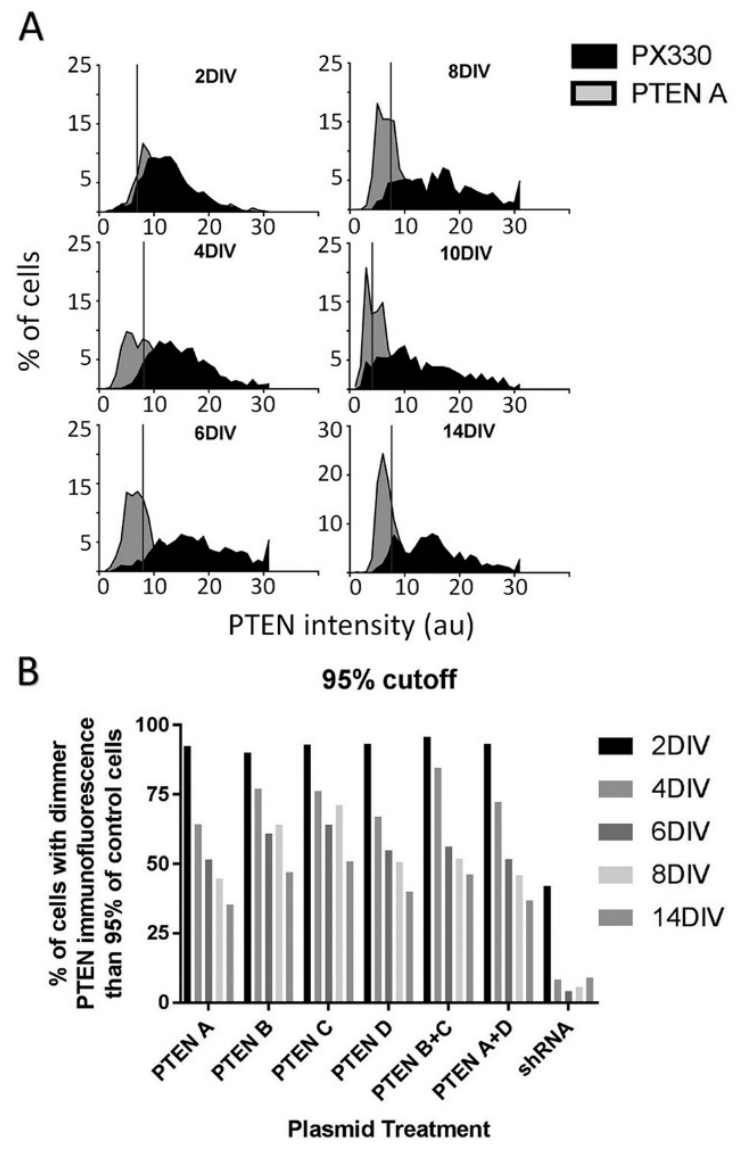

Fig. 3. Four distinct anti-PTEN CRISPR constructs display similar timecourse of protein knockdown, which is delayed compared to shRNA-mediated knockdown. P3-P5 cortical neurons were co-transfected with EGFP reporter and plasmid co-expressing spCas9 and one of four distinct sgRNA sequences targeting PTEN, or mixtures of two sequences. In parallel, neurons were also transfected with anti-PTEN shRNA. Between 2 and 14 days later, cells were fixed and immunostained for PTEN protein, and Cellomics automated microscopy was 
used to quantify immunofluorescence in EGFP + cells. (A) shows example histograms comparing PTEN immunofluorescence in populations of neurons transfected with anti-PTEN sgRNA and non-targeting control. At two days post-transfection the populations mostly overlap, but in subsequent days the percent of cells with low PTEN expression increases in the sgRNA treatment. Vertical lines indicate a threshold of PTEN signal set by the dimmest $5 \%$ of control cells. (B) quantifies the percent of EGFP + cells with PTEN intensity below a the $5 \%$ threshold at the same timepoint. At two days post-transfection, single and mixed sgRNA-treated populations display only $5 \%$ of cells below this threshold, while $>50 \%$ of shRNA-treated cells fall below threshold. By six days, between 40 and $50 \%$ of sgRNA-treated cells fall below threshold. More than 500 cells were analyzed in each treatment at each timepoint.

\subsection{Functional testing CRISPR-mediated phenotype in an HCS workflow} Having established effective CRISPR-mediated protein reduction in primary neurons, we next asked whether this approach could yield reproducible effects on neurite outgrowth using approaches compatible with HCS screening. Previous screens have utilized plasmid electroporation and culture in multi-well format, followed by automated image acquisition and quantification of neurite outgrowth with Cellomics instrumentation (Blackmore et al., 2010, Simpson et al., 2015). This endpoint analysis is typically performed no more than three days in culture, after which time extensive overlap of neurites makes cell-based quantification very difficult. Although the previous timecourse data suggested it

might be unlikely that phenotypic effects of CRISPR-mediated knockdown would be detectable within three days of transfection, we nevertheless tested the effects of anti-PTEN sgRNA using this lab standard protocol. As before, P4 cortical neurons were transfected with PX330-based plasmids encoding spCas9 and non-target, anti-PTEN, or anti-NeuN sgRNA as well as an EGFP reporter at a 1:4 ratio. Neurons were then cultured on PDL/laminin substrate for three days, fixed and stained with neuron-specific BIII tubulin, and neurite lengths quantified in transfected neurons. As shown in Fig. 4, neurite length in cells transfected with anti-PTEN sgRNA did not differ significantly from anti-NeuN or non-target controls.
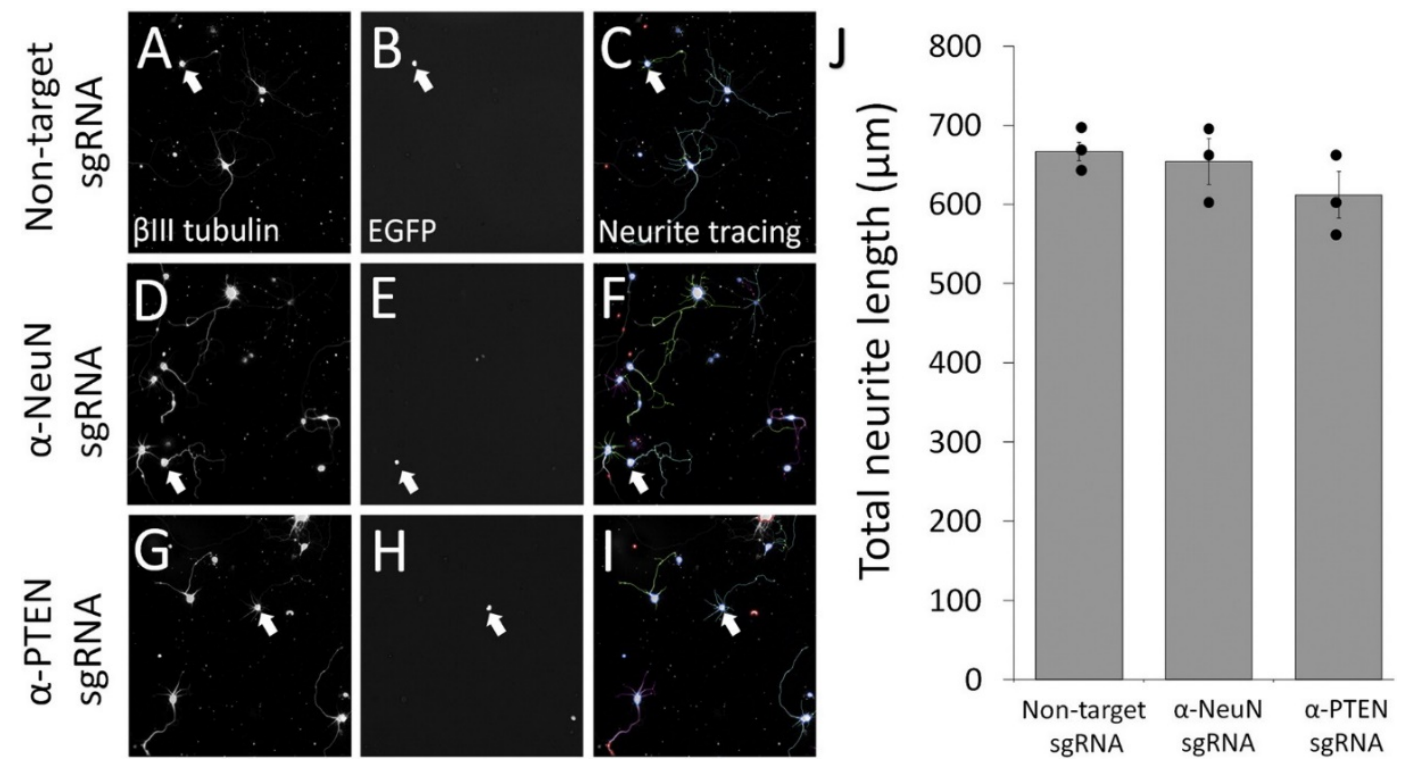

Fig. 4. Short-term treatment with anti-PTEN CRISPR does not affect neurite length in primary cortical neurons. P3-P5 cortical neurons were transfected with plasmid DNA co-expressing spCas9, anti-NeuN, anti-PTEN or nontargeting sgRNA, along with plasmid encoding EGFP reporter. (A-I) Example images from ThermoScientific Cellomics CX5 high content screening platform. Neurons are identified by $\beta$ III-tubulin $(A, D, G)$ transfection by EGFP (arrows, B, E, H), and neurite are traced $(C, F, I)$ using $\beta$ III-tubulin fluorescence $(J)$ Quantification of total 
neurite length shows no significant differences between any groups $(p>0.05$, matched pair $t$-test with Bonferonni's correction). $N>100$ cells per group in three replicate experiments.

One possible explanation for these negative results is that during the first three days post-transfection the degree of protein reduction was insufficient to affect neurite lengths. To test this possibility we adopted a strategy of maintaining longer term, high-density cultures, followed by replating. As before, P3-5 cortical neurons were transfected in multi-well format, but were plated and maintained at a relatively high density $(100,000$ cells plated per well in 24 -well format) for seven days. As a positive control, additional cells were transfected with plasmid to overexpress Jun, shown previously to enhance neurite outgrowth in this cell type (Lerch et al., 2014, Simpson et al., 2015). FuDR was added to the media to block glial proliferation, resulting in $>95 \%$ pure neuronal cultures. After seven days, neurons were dissociated with Accutase and replated at a density of 10,000 cells per well on $\mathrm{PDL} /$ laminin substrates. After $24 \mathrm{~h}$ of neurite extension the replated neurons were fixed, stained with BIII tubulin, and analyzed with Cellomics instrumentation as above (Fig. 5A-I). Although this procedure resulted in significant cell death, several hundred transfected neurons survived and extended neurites that were readily quantified. JUN overexpression enhanced neurite outgrowth by an average of $33.5 \%$ ( $\pm 12.8 \%$ SEM) across three replicate experiments, similar to the $26.2 \%$ ( $\pm 6.9 \%$ SEM) seen in previous short-term screening experiments (Fig. 5J) (Simpson et al., 2015). These data confirm the sensitivity of this replating approach for detection of known growth phenotypes. In replated neurons treated with anti-PTEN sgRNA, neurite lengths were significantly elevated compared to non-targeted controls across four replicate experiments (136.8 \pm 8.0\% SEM, $p<0.01$, ANOVA with post-hoc Dunnett's) (Fig. 5J). Immunohistochemistry for PTEN protein in the replated neurons confirmed effective knockdown, as $75 \%$ of cells fell below the threshold set by the dimmest $5 \%$ of control neurons (Supplementary Fig. 2). These data indicate that reduction of PTEN expression in early postnatal cortical neurons results in increased neurite lengths. 


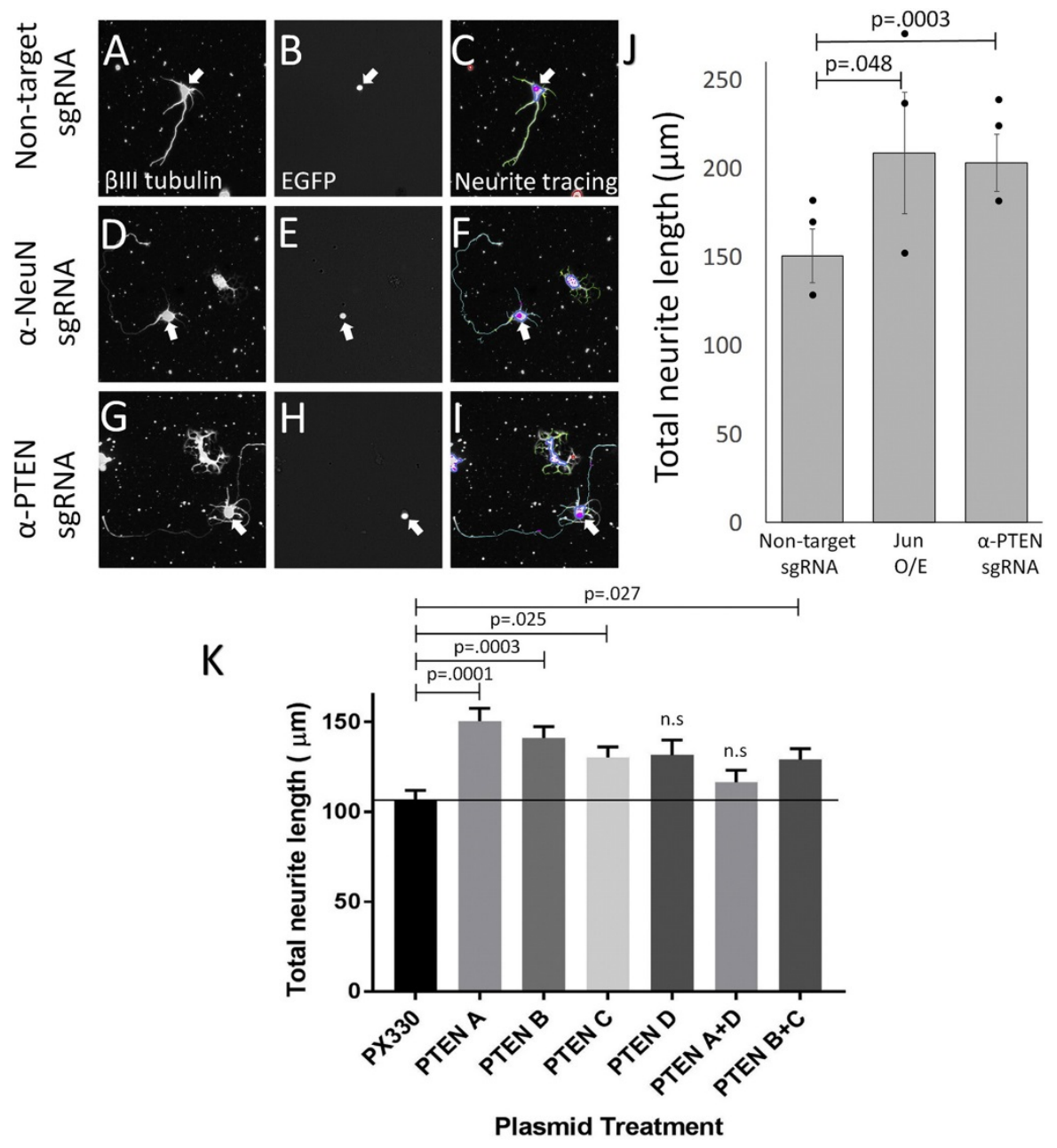

Fig. 5. CRISPR-mediated knockdown of PTEN increases neurite length at a 7DIV timepoint. P3-P5 cortical neurons were transfected with plasmid DNA expressing Jun or spCas9 and sgRNA (anti-PTEN or non-targeting sgRNA), along with plasmid EGFP reporter. (A-I) show example images from ThermoScientific Cellomics CX5 high content screening platform. The automated microscope identifies neurons by $\beta$ III-tubulin $(A, D, G)$ and transfection by EGFP (arrows) (B, E, H). Cellinsight Cell Analysis software then automatically traces neurites (C, F, I) using the $\beta I I I-t u b u l i n$ fluorescence. Compared to non-targeted sgRNA control $(A, B, C$,$) , increases in neurite$ length of transfected cells are apparent after Jun overexpression (D, E, F) or PTEN knockout $(G, H, I)$. (J) Quantification of total neurite length shows significant increases in neurons treated with Jun overexpression and anti-PTEN sgRNA ( $p<0.05$, matched pair $t$-test with Bonferonni's correction). $N \geq 100$ cells in 3 experimental replicates. (K) In a separate experiment, neurons were transfected with four distinct anti-PTEN sgRNA sequences, or pairwise combinations, maintained 7DIV, and then replated. $24 \mathrm{~h}$ later, Cellomics-based quantification showed that three constructs caused significant increases in total neurite length, and the fourth showed a similar trend. $\mathrm{p}<0.05$, ANOVA with post-hoc Dunnett's, $\mathrm{N}>500$ cells per treatment.

It is possible that increased neurite outgrowth produced by treatment with anti-PTEN CRISPR sequences could result from off-target effects. Although off-target mutations can be detected through approaches like next-generation sequencing, a more practical solution in a screening experiment is to employ multiple targeting sequences against distinct regions of the same gene. Because different sgRNA constructs are unlikely to affect common off-target sequences, consistency of the evoked phenotype increases confidence that on-target knockdown explains the observed effects. Indeed, we found that three of the four validated anti-PTEN sgRNA sequences significantly increased neurite length compared to non-specific sgRNA ( $p<0.05$, ANOVA with post-hoc Dunnett's), and the forth 
sequence displaying a similar trend (Fig. 5K). Thus it is most likely that CRISPR-mediated knockdown of PTEN protein, as opposed to off-target effects, was responsible for the increased neurite length.

Importantly, these data also provide positive control sequences for CRISPR-mediated effects on neurite outgrowth, laying critical groundwork for the establishment of CRISPR-based screening approaches in primary neurons.

\section{Discussion}

We have assessed the suitability of CRISPR-based genome editing for use in phenotypic screening in primary neurons. Using cultured cortical neurons, we have established the time course and efficacy of CRISPR knock down for two proteins, NeuN and PTEN. We also established a replating technique for postnatal cortical neurons in order to assay neurite outgrowth when an extended period in culture is required. Finally, we show that CRISPR-based knockout of PTEN, delivered by electroporation in multiwell format, causes an increase in neurite outgrowth that is readily detectable by an automated screening platform. Overall, these results demonstrate the feasibility of performing a CRISPR-based high content knockout screen.

\subsection{The role of PTEN in early postnatal neurite outgrowth}

PTEN, an upstream inhibitor of PI3K signaling and the mTOR pathway, was selected for this validation study because it has been shown to act in a variety of cell types as a negative regulator of axon extension. For example, transgenic knockout and shRNA-mediated knockdown of PTEN cause increased regenerative axon growth in retinal ganglion cells (Park et al., 2008, Yungher et al., 2015), corticospinal tract neurons (Liu et al., 2010, Zukor et al., 2013), rubrospinal neurons (Geoffroy et al., 2016), and dorsal root ganglion neurons (Christie et al., 2010). The timing of knockdown appears to affect the rate of axon extension triggered by PTEN ablation, with stronger regenerative responses observed when PTEN is pre-ablated early in postnatal development and injuries performed after maturation (Geoffroy et al., 2016). Indeed, despite the clear role for PTEN in limiting axon growth in adult CNS neurons, it was previously unclear whether PTEN is similarly inhibitory in the cellular context of immature neurons, which possess a higher innate growth ability and lower levels of endogenous PTEN. The current data clearly indicate that PTEN is present in cortical neurons during early postnatal periods, and that PTEN ablation increases total neurite extension.

\subsection{Challenges and unanswered questions}

An important question for any knockdown approach is the degree of specificity. In the case of CRISPR, knockout of off-target proteins by sgRNA could potentially produce phenotypic changes, leading to false positive screening results. Cleavage efficiency by CRISPR is strongly reduced by mismatches in the 20 nt sgRNA/DNA binding site, particularly if multiple mismatches are located in PAM-proximal region (Hsu et al., 2013). Publically available algorithms now exist to scan genomes and identify sgRNA sequences with minimized potential for off-target cleavage; the sequences used here utilized the method of Hsu et al. (2013). Nevertheless, the potential remains for off-target DNA cleavage, and a variety of techniques have been developed in non-neuronal cells to detect them (reviewed in Wu et al., 2014). For example, potential off-target sequences in the genome can be PCR-amplified and sequenced or analyzed by mismatch-specific nucleases (e.g. Surveyor). The most comprehensive approach is to perform whole genome sequencing of cell populations clonally derived from single sgRNA-treated cells (Smith et al., 2014, Veres et al., 2014). Genome-wide sequencing is likely not feasible in individual postmitotic neurons, and even PCR-based analysis of predicted off-target cleavage is likely impractical for most screening projects. Thus, the best safeguard against off-target, false-positive hits will likely be to 
test multiple sgRNA sequences against the same protein target, similar to the strategy adopted by previous RNAi screens (Nieland et al., 2014, Sharma et al., 2013, Welsbie et al., 2013). Importantly, our data indicate that in the case of PTEN knockdown, three of four sgRNA targeting sequences produced a significant increase in neurite length. Setting requirements for consistent phenotype across multiple targeting sequences greatly increases the probability that on-target effects are responsible.

Several groups have shown that RNAi approaches can cause non-specific effects through an innate immune response resulting in the expression of interferon proteins (Bridge et al., 2003, Sledz et al., 2003). Of particular relevance, lentiviral-mediated delivery of shRNA to primary neurons has been shown to elevate expression of interferon-stimulated genes such as Oas1, with attendant decreases in viability and neurite outgrowth (Bauer et al., 2009, Hutson et al., 2012). Incorporation of microRNA flanking sequences and designs that prevent the formation of perfect double stranded RNA helixes have been used to alleviate this interferon response (Bauer et al., 2009, Cullen, 2006). It is unknown whether Cas 9 and sgRNA induce a similar response, although the single-stranded nature of the sgRNA may make it less likely. Nevertheless, in future work it will be critical to directly test for interferon responses and other potential non-specific effects of CRISPR in primary neurons.

Another potential challenge is presented by the finding that CRISPR-mediated knockdown can take up to six days to affect the majority of transfected cells. After plasmid transfection, protein knockout can occur only after 1) production of Cas9 enzyme and sgRNA 2) Cas9-mediated cleavage at both genomic copies of PTEN and 3) clearance of existing protein pools. Which step contributes most to the six-day delay? We observe plasmid-driven expression of reporter proteins within $16 \mathrm{~h}$ of transfection, making it unlikely that delayed expression of Cas9 protein is a major culprit. Similarly, the half-life of PTEN has been estimated at about $7.5 \mathrm{~h}$ (Trotman et al., 2007), and using shRNA we observe knockdown that is strong at 2DIV and nearly complete by 4DIV (Fig. 3), consistent with previous use of this shRNA targeting sequence (Yungher et al., 2015). Thus, a slow rate of PTEN turnover is also unlikely to explain the delay. Combined, these observations suggest a model in which DNA cleavage by Cas9 is a stochastic event that affects an expanding fraction of the population over the course of several days. Expanded experimentation is needed to confirm that a six day lag to knockdown is a general feature of the CRISPR strategy in primary neurons. Nevertheless, it is notable that we observed very similar timecourses for five distinct sgRNAs against two protein targets. Thus potential users should be alert to the possibility that screens involving CRISPR manipulation of primary neurons may require approximately a week in culture to achieve protein knockdown in a majority of transfected cells, followed by additional time in culture for the phenotypic consequences to manifest.

One consideration is that the timing of knockout may vary between individual cells (i.e. Cas9 cleavage may have affected some cells at 2DIV, others not until 6DIV). This range of time-since-knockout could act as a source of phenotypic variability. In addition, the lag between transfection and protein knockout may present a technical hurdle for some endpoint measurements, including screens of neurite outgrowth considered here. Indeed, we empirically tested and ruled out the possibility that CRISPR-based approach might be compatible with previous HCS workflows, in which neurite outgrowth was quantified at a 3DIV timepoint (Blackmore et al., 2010). We therefore adopted a replating technique, similar to procedures that we and others have used previously to disentangle dorsal root ganglion (DRG) neurons after extended culture (Simpson et al., 2015, Zou et al., 2009). This approach is feasible but introduces an extra step into the workflow and results in significant cell death, which increases the number of cells needed for the initial transfection step. In addition, the replating procedure likely introduces an increase in variability in subsequent neurite outgrowth, potentially reducing screen sensitivity. Indeed, when compared to prior data from non-replated neurons, we find 
a significant increase in variance of neurite length (standard deviation approximately $25 \%$ higher, $F$-test $p<0.01$ ). Despite these caveats, however, this approach did produce consistent enhancement of neurite outgrowth after JUN overexpression and CRISPR-mediated PTEN knockout. The establishment of a consistent positive control is a promising step toward the use of CRISPR knockout in neurite outgrowth screens.

Now that we have established positive controls it will be important to reduce variability and increase the sensitivity of future screens. One source of variability is imperfect co-transfection between separate Cas 9 and EGFP plasmids, although our previous data predict a $>90 \%$ co-expression at the 1:4 ratios used here (Blackmore et al., 2010). The PX330 vector we tested here does not include a reporter, and the large size of Cas9 creates challenges for reporter co-expression from a single plasmid. As a potential future solution, CRISPR technology allows for targeted homology-directed repair (HDR) (Cong et al., 2013). In this way a repair template containing either a fluorescent reporter or an antibiotic selection marker could be inserted when a target gene is nicked by Cas9. In principle, this approach would specifically identify cells affected by Cas9 enzyme. Challenges to this strategy include the fact that HDR has an extremely low efficiency, and the difficulty in distinguishing homozygous from heterozygous insertion, which could leave one copy of the target gene intact (Maruyama et al., 2015). Nevertheless, as CRISPR technologies rapidly evolve, HDR-based strategies may become more practical in a HCS environment.

\subsection{Future prospects for CRISPR-based screening in primary neurons}

One factor that may favor the adoption of CRISPR-based screening is the simplicity of creating effective reagents. In the current study, publically available algorithms were used to design targeting sequences ranked on the basis of specificity and efficacy. Following procedures described in Cong et al. (2013), short oligonucleotides were synthesized, annealed, and ligated into PX330 plasmid which co-expresses Cas9 and sgRNA. This process proved to be simple and highly economical; two features that make it portable to high content screens. This raises the prospect that large-scale, custom-designed CRISPR knockout libraries could be quickly and inexpensively created.

A second potential advantage is the prospect of multiplex screening, e.g. simultaneous knockdown of multiple target genes. It has been shown that in the presence of multiple sgRNAs, Cas nucleases can cleave diverse DNA sequences in the same cell (Cong et al., 2013). This strategy has been used to achieve multiplex knockdown in a variety of systems, including zebrafish, mouse embryonic stem cells, human immortalized myoblasts, HEK293T cells, and yeast (Jakociunas et al., 2015, Jao et al., 2013, Kabadi et al., 2014, Ousterout et al., 2015, Yang et al., 2013). Notably, simultaneous knockdown of three proteins (DNMT1, DNMT3a, and DNMT3b) was recently demonstrated using a CRISPR approach in neurons (Swiech et al., 2015). Combined with the high economy of sgRNA production these studies pave the way for a combinatorial screening approach in which multiple genes are concurrently targeted for knockout.

Third, future screening projects could take advantage of the precision of CRISPR gene targeting without the ensuing DNA disruption. This is because Cas nucleases can be rendered catalytically inactive and unable to cleave DNA while still maintaining the ability to bind specific genomic loci under the control of user-designed sgRNA. One simple example is the fusion of the transcriptional activation domain VP64 to dCas9 to create a tool for targeted upregulation of specific genes (Perez-Pinera et al., 2013). The practical applications of targeted gene manipulation are nearly limitless and include the possibility of performing simultaneous, combinatorial knockout and overexpression screening. 
Finally, CRISPR-mediated knockout has now been demonstrated to be highly effective in CNS neurons in vivo. Although the large size of the spCas9 enzyme can present challenges to viral delivery, strategies to circumvent this problem are being developed. For instance, the use of smaller Cas 9 family members or transgenic animals that conditionally express spCas9 are proving to be effective (Cong et al., 2013, Ran et al., 2015). Viral delivery of multiple sgRNA may therefore represent a rapid and flexible alternative to current transgenic approaches to achieving simultaneous knockout of multiple genes. Moreover, transgenic animals with constitutively expressed Cas9 will likely be useful as a source of cells for in vitro screening by simplifying sgRNA delivery and perhaps accelerating the onset of knockdown by eliminating the lag between transfection and Cas9 expression. It is clear that axon regeneration is regulated by many interacting genes and combinatorial gene manipulations have proven most effective in promoting growth (Belin et al., 2015, Jin et al., 2015, Sun et al., 2011). Thus the prospect exists that CRISPR-based approaches may accelerate both in vitro discovery of gene interactions that regulate axon growth, and the transition to combinatorial testing of lead hits in vivo. Overall it is clear that CRISPR-based approaches have enormous potential to expand understanding of interactive gene function in a variety of cell types. In this context, the current demonstration of CRISPR efficacy and compatibility with high content screening in cultured CNS neurons is a promising indicator that this potential can be harnessed for neuroscience research.

The following are the supplementary data related to this article.

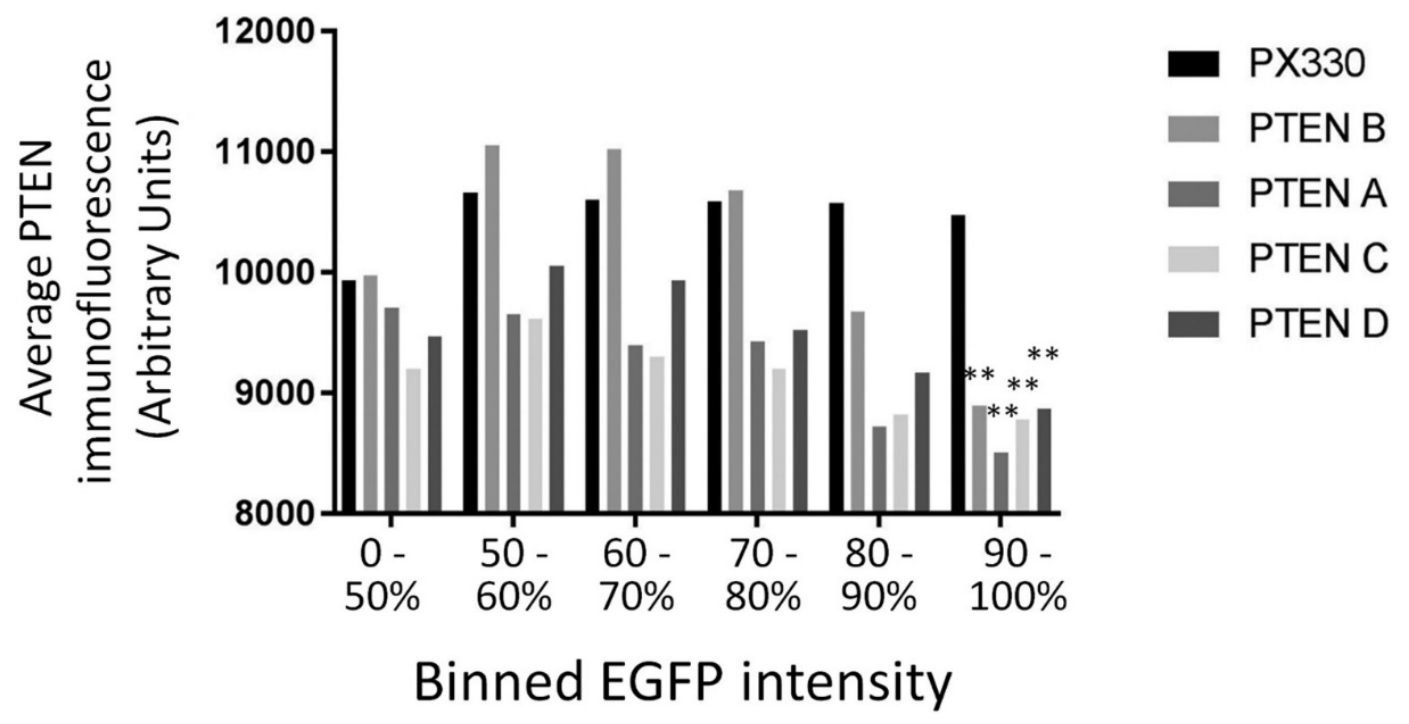

Supplementary Fig. 1. The degree of CRISPR-mediated knockdown of PTEN correlates with EGFP-reporter intensity. P3-P5 cortical neurons were co-transfected with EGFP reporter plasmid and plasmid co-expressing spCas9 and one of four distinct sgRNA sequences targeting PTEN, or non-targeting sgRNA. At 14 days posttransfection cells were fixed and immunostained for PTEN and Cellomics automated microscopy quantified immunofluorescence and EGFP reporter intensity. Then, the PTEN fluorescence of all cells was binned by EGFP intensity to determine the relationship between the fluorescence of the transfection marker and the level of PTEN knockdown. In all anti-PTEN sgRNA treatments $10 \%$ of cells with the brightest EGFP signal show a large and consistent reduction in PTEN fluorescence compared to the non-targeting control treatment $(p<0.001$, ANOVA with post-hoc Dunnett's). 

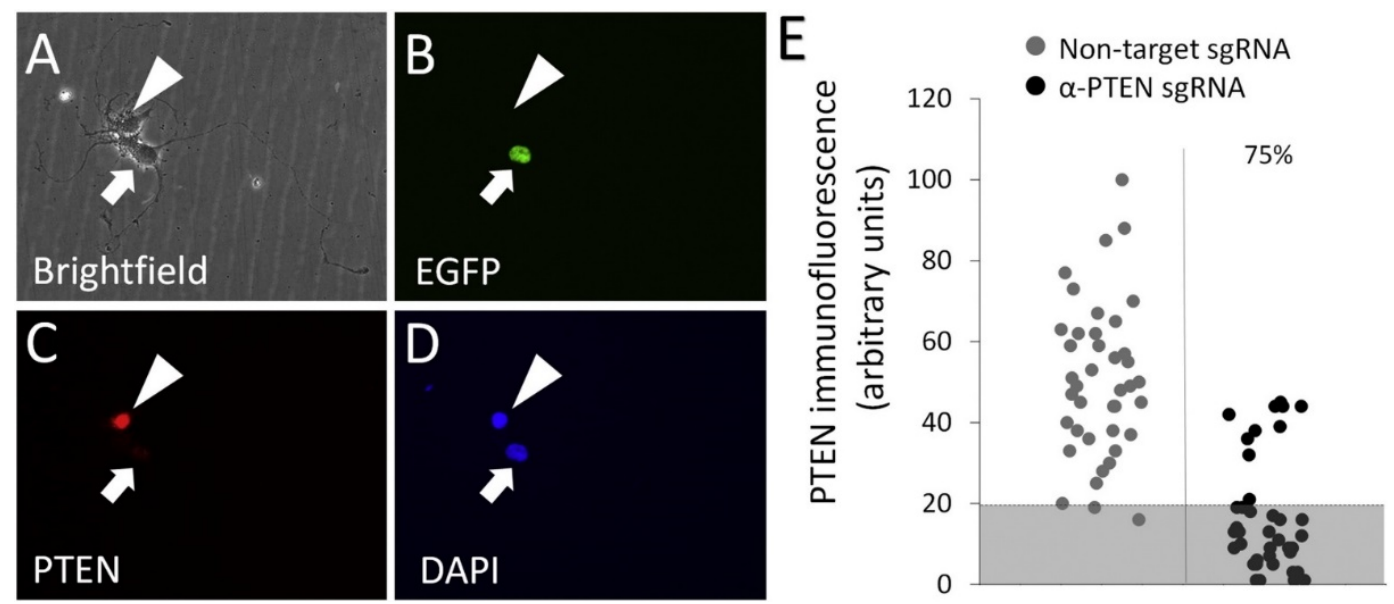

Supplementary Fig. 2. CRISPR-mediated decreases in PTEN expression persist following replating at 7DIV. P3-P5 cortical neurons were transfected with plasmid DNA co-expressing spCas9 and anti-PTEN or non-targeting sgRNA, along with plasmid EGFP reporter. (A-D) Show example image of replated cortical neurons. (A) Brightfield shows two neurons extending neurites $24 \mathrm{~h}$ following replating. (B) EGFP identifies transfection (arrow). (C) Immunohistochemistry detects PTEN protein in an EGFP-negative neuron (arrowhead), but not in a transfected neighbor (arrow). (D) DAPI staining identifies both objects as nuclei containing cells (E) Each dot represents PTEN immunofluorescence intensity in a single transfected (EGFP +) neuron at 7DIV plus 24 h postreplating. Grey boxes indicate a threshold set by the dimmest $5 \%$ of control-transfected neurons, and the percent of anti-PTEN cells that fall below that threshold is quantified above. Anti-PTEN sgRNA induces a significant reduction in the population average PTEN intensity $\left(p=2.5 \times 10^{-13}\right.$, unpaired $t$-test), with $75 \%$ of cells below threshold. $\mathrm{N}=40$ cells per group.

\section{Conflict of interest}

None.

\section{Acknowledgments}

This work was supported by NIHR21NS093278 and the Bryon Riesch Paralysis Foundation.

\section{References}

An et al., 2014 M.C. An, R.N. O'Brien, N. Zhang, B.N. Patra, M. De La Cruz, A. Ray, L.M. Ellerby. Polyglutamine disease modeling: epitope based screen for homologous recombination using CRISPR/Cas9 system. PLoS Curr., 6 (2014).

Bauer et al., 2009 M. Bauer, N. Kinkl, A. Meixner, E. Kremmer, M. Riemenschneider, H. Forstl, T. Gasser, M. Ueffing. Prevention of interferon-stimulated gene expression using microRNAdesigned hairpins. Gene Ther., 16 (2009), pp. 142-147.

Belin et al., 2015 S. Belin, H. Nawabi, C. Wang, S. Tang, A. Latremoliere, P. Warren, H. Schorle, C. Uncu, C.J. Woolf, Z. He, J.A. Steen. Injury-induced decline of intrinsic regenerative ability revealed by quantitative proteomics. Neuron, 86 (2015), pp. 1000-1014.

Blackmore, 2012 M.G. Blackmore. Molecular control of axon growth: insights from comparative gene profiling and high-throughput screening. Int. Rev. Neurobiol., 105 (2012), pp. 39-70.

Blackmore et al., 2010 M.G. Blackmore, D.L. Moore, R.P. Smith, J.L. Goldberg, J.L. Bixby, V.P. Lemmon. High content screening of cortical neurons identifies novel regulators of axon growth. Mol. Cell. Neurosci., 44 (2010), pp. 43-54. 
Blackmore et al., 2012 M.G. Blackmore, Z. Wang, J.K. Lerch, D. Motti, Y.P. Zhang, C.B. Shields, J.K. Lee, J.L. Goldberg, V.P. Lemmon, J.L. Bixby. Kruppel-like factor 7 engineered for transcriptional activation promotes axon regeneration in the adult corticospinal tract. Proc. Natl. Acad. Sci. U. S. A., 109 (2012), pp. 7517-7522.

Bridge et al., 2003 A.J. Bridge, S. Pebernard, A. Ducraux, A.L. Nicoulaz, R. Iggo. Induction of an interferon response by RNAi vectors in mammalian cells. Nat. Genet., 34 (2003), pp. 263-264.

Buchser et al., 2006 W.J. Buchser, J.R. Pardinas, Y. Shi, J.L. Bixby, V.P. Lemmon. 96-well electroporation method for transfection of mammalian central neurons. BioTechniques, 41 (2006), pp. 619-624.

Buchser et al., 2012 W.J. Buchser, R.P. Smith, J.R. Pardinas, C.L. Haddox, T. Hutson, L. Moon, S.R. Hoffman, J.L. Bixby, V.P. Lemmon. Peripheral nervous system genes expressed in central neurons induce growth on inhibitory substrates. PLoS One, 7 (2012), Article e38101.

Chen et al., 2015 S. Chen, N.E. Sanjana, K. Zheng, O. Shalem, K. Lee, X. Shi, D.A. Scott, J. Song, J.Q. Pan, R. Weissleder, H. Lee, F. Zhang, P.A. Sharp. Genome-wide CRISPR screen in a mouse model of tumor growth and metastasis. Cell, 160 (2015), pp. 1246-1260.

Christie et al., 2010 K.J. Christie, C.A. Webber, J.A. Martinez, B. Singh, D.W. Zochodne. PTEN inhibition to facilitate intrinsic regenerative outgrowth of adult peripheral axons. J. Neurosci., 30 (2010), pp. 9306-9315.

Cong et al., 2013 L. Cong, F.A. Ran, D. Cox, S. Lin, R. Barretto, N. Habib, P.D. Hsu, X. Wu, W. Jiang, L.A. Marraffini, F. Zhang. Multiplex genome engineering using CRISPR/Cas systems. Science, 339 (2013), pp. 819-823.

Cullen, 2006 B.R. Cullen. Induction of stable RNA interference in mammalian cells. Gene Ther., 13 (2006), pp. 503-508.

Doench et al., 2014 J.G. Doench, E. Hartenian, D.B. Graham, Z. Tothova, M. Hegde, I. Smith, M. Sullender, B.L. Ebert, R.J. Xavier, D.E. Root. Rational design of highly active sgRNAs for CRISPRCas9-mediated gene inactivation. Nat. Biotechnol., 32 (2014), pp. 1262-1267.

Ebert et al., 2008 B.L. Ebert, J. Pretz, J. Bosco, C.Y. Chang, P. Tamayo, N. Galili, A. Raza, D.E. Root, E. Attar, S.R. Ellis, T.R. Golub. Identification of RPS14 as a 5q-syndrome gene by RNA interference screen. Nature, 451 (2008), pp. 335-339.

Geoffroy et al., 2016 C.G. Geoffroy, B.J. Hilton, W. Tetzlaff, B. Zheng. Evidence for an age-dependent decline in axon regeneration in the adult mammalian central nervous system. Cell Rep., 15 (2016), pp. 238-246.

Hsu et al., 2013 P.D. Hsu, D.A. Scott, J.A. Weinstein, F.A. Ran, S. Konermann, V. Agarwala, Y. Li, E.J. Fine, X. Wu, O. Shalem, T.J. Cradick, L.A. Marraffini, G. Bao, F. Zhang. DNA targeting specificity of RNA-guided Cas9 nucleases. Nat. Biotechnol., 31 (2013), pp. 827-832.

Hutson et al., 2012 T.H. Hutson, E. Foster, J.M. Dawes, R. Hindges, R.J. Yanez-Munoz, L.D. Moon. Lentiviral vectors encoding short hairpin RNAs efficiently transduce and knockdown LINGO-1 but induce an interferon response and cytotoxicity in central nervous system neurons. J. Gene Med., 14 (2012), pp. 299-315.

Incontro et al., 2014 S. Incontro, C.S. Asensio, R.H. Edwards, R.A. Nicoll. Efficient, complete deletion of synaptic proteins using CRISPR. Neuron, 83 (2014), pp. 1051-1057.

Jakociunas et al., 2015 T. Jakociunas, I. Bonde, M. Herrgard, S.J. Harrison, M. Kristensen, L.E. Pedersen, M.K. Jensen, J.D. Keasling. Multiplex metabolic pathway engineering using CRISPR/Cas9 in Saccharomyces cerevisiae. Metab. Eng., 28 (2015), pp. 213-222.

Jao et al., 2013 L.E. Jao, S.R. Wente, W. Chen. Efficient multiplex biallelic zebrafish genome editing using a CRISPR nuclease system. Proc. Natl. Acad. Sci. U. S. A., 110 (2013), pp. 13904-13909. 
Jin et al., 2015 D. Jin, Y. Liu, F. Sun, X. Wang, X. Liu, Z. He. Restoration of skilled locomotion by sprouting corticospinal axons induced by co-deletion of PTEN and SOCS3. Nat. Commun., 6 (2015), p. 8074.

Kabadi et al., 2014 A.M. Kabadi, D.G. Ousterout, I.B. Hilton, C.A. Gersbach. Multiplex CRISPR/Cas9based genome engineering from a single lentiviral vector. Nucleic Acids Res., 42 (2014), Article e147.

Kunin et al., 2007 V. Kunin, R. Sorek, P. Hugenholtz. Evolutionary conservation of sequence and secondary structures in CRISPR repeats. Genome Biol., 8 (2007), p. R61.

Lerch et al., 2014 J.K. Lerch, Y.R. Martinez-Ondaro, J.L. Bixby, V.P. Lemmon. cJun promotes CNS axon growth. Mol. Cell. Neurosci., 59 (2014), pp. 97-105.

Liu et al., 2010 K. Liu, Y. Lu, J.K. Lee, R. Samara, R. Willenberg, I. Sears-Kraxberger, A. Tedeschi, K.K. Park, D. Jin, B. Cai, B. Xu, L. Connolly, O. Steward, B. Zheng, Z. He. PTEN deletion enhances the regenerative ability of adult corticospinal neurons. Nat. Neurosci., 13 (2010), pp. 1075-1081.

Maruyama et al., 2015 T. Maruyama, S.K. Dougan, M.C. Truttmann, A.M. Bilate, J.R. Ingram, H.L. Ploegh. Increasing the efficiency of precise genome editing with CRISPR-Cas9 by inhibition of nonhomologous end joining. Nat. Biotechnol., 33 (2015).

Meyer-Franke et al., 1995 A. Meyer-Franke, M.R. Kaplan, F.W. Pfrieger, B.A. Barres. Characterization of the signaling interactions that promote the survival and growth of developing retinal ganglion cells in culture. Neuron, 15 (1995), pp. 805-819.

Moffat et al., 2006 J. Moffat, D.A. Grueneberg, X. Yang, S.Y. Kim, A.M. Kloepfer, G. Hinkle, B. Piqani, T.M. Eisenhaure, B. Luo, J.K. Grenier, A.E. Carpenter, S.Y. Foo, S.A. Stewart, B.R. Stockwell, N. Hacohen, W.C. Hahn, E.S. Lander, D.M. Sabatini, D.E. Root. A lentiviral RNAi library for human and mouse genes applied to an arrayed viral high-content screen. Cell, 124 (2006), pp. 12831298.

Nieland et al., 2014 T.J. Nieland, D.J. Logan, J. Saulnier, D. Lam, C. Johnson, D.E. Root, A.E. Carpenter, B.L. Sabatini. High content image analysis identifies novel regulators of synaptogenesis in a high-throughput RNAi screen of primary neurons. PLoS One, 9 (2014), Article e91744.

Ohtake et al., 2015 Y. Ohtake, U. Hayat, S. Li. PTEN inhibition and axon regeneration and neural repair. Neural Regen. Res., 10 (2015), pp. 1363-1368.

Ousterout et al., 2015 D.G. Ousterout, A.M. Kabadi, P.I. Thakore, W.H. Majoros, T.E. Reddy, C.A. Gersbach. Multiplex CRISPR/Cas9-based genome editing for correction of dystrophin mutations that cause Duchenne muscular dystrophy. Nat. Commun., 6 (2015), p. 6244.

Park et al., 2008 K.K. Park, K. Liu, Y. Hu, P.D. Smith, C. Wang, B. Cai, B. Xu, L. Connolly, I. Kramvis, M. Sahin, Z. He. Promoting axon regeneration in the adult CNS by modulation of the PTEN/mTOR pathway. Science, 322 (2008), pp. 963-966.

Perez-Pinera et al., 2013 P. Perez-Pinera, D.D. Kocak, C.M. Vockley, A.F. Adler, A.M. Kabadi, L.R. Polstein, P.I. Thakore, K.A. Glass, D.G. Ousterout, K.W. Leong, F. Guilak, G.E. Crawford, T.E. Reddy, C.A. Gersbach. RNA-guided gene activation by CRISPR-Cas9-based transcription factors. Nat. Methods, 10 (2013), pp. 973-976.

Platt et al., 2014 R.J. Platt, S. Chen, Y. Zhou, M.J. Yim, L. Swiech, H.R. Kempton, J.E. Dahlman, O. Parnas, T.M. Eisenhaure, M. Jovanovic, D.B. Graham, S. Jhunjhunwala, M. Heidenreich, R.J. Xavier, R. Langer, D.G. Anderson, N. Hacohen, A. Regev, G. Feng, P.A. Sharp, F. Zhang. CRISPRCas9 knockin mice for genome editing and cancer modeling. Cell, 159 (2014), pp. 440-455.

Ran et al., 2015 F.A. Ran, L. Cong, W.X. Yan, D.A. Scott, J.S. Gootenberg, A.J. Kriz, B. Zetsche, O. Shalem, X. Wu, K.S. Makarova, E.V. Koonin, P.A. Sharp, F. Zhang. In vivo genome editing using Staphylococcus aureus Cas9. Nature, 520 (2015), pp. 186-191. 
Root et al., 2006 D.E. Root, N. Hacohen, W.C. Hahn, E.S. Lander, D.M. Sabatini. Genome-scale loss-offunction screening with a lentiviral RNAi library. Nat. Methods, 3 (2006), pp. 715-719.

Schmid-Burgk et al., 2016 J.L. Schmid-Burgk, D. Chauhan, T. Schmidt, T.S. Ebert, J. Reinhardt, E. Endl, V. Hornung. A genome-wide CRISPR (clustered regularly interspaced short palindromic repeats) screen identifies NEK7 as an essential component of NLRP3 inflammasome activation. J. Biol. Chem., 291 (2016), pp. 103-109.

Sharma et al., 2013 K. Sharma, S.Y. Choi, Y. Zhang, T.J. Nieland, S. Long, M. Li, R.L. Huganir. Highthroughput genetic screen for synaptogenic factors: identification of LRP6 as critical for excitatory synapse development. Cell Rep., 5 (2013), pp. 1330-1341.

Simpson et al., 2015 M.T. Simpson, I. Venkatesh, B.L. Callif, L.K. Thiel, D.M. Coley, K.N. Winsor, Z. Wang, A.A. Kramer, J.K. Lerch, M.G. Blackmore. The tumor suppressor HHEX inhibits axon growth when prematurely expressed in developing central nervous system neurons. Mol. Cell. Neurosci., 68 (2015), pp. 272-283.

Sledz et al., 2003 C.A. Sledz, M. Holko, M.J. de Veer, R.H. Silverman, B.R.G. Williams. Activation of the interferon system by short-interfering RNAs. Nat. Cell Biol., 5 (2003), pp. 834-839.

Smith et al., 2014 C. Smith, A. Gore, W. Yan, L. Abalde-Atristain, Z. Li, C. He, Y. Wang, R.A. Brodsky, K. Zhang, L. Cheng, Z. Ye. Whole-genome sequencing analysis reveals high specificity of CRISPR/Cas9 and TALEN-based genome editing in human iPSCs. Cell Stem Cell, 15 (2014), pp. 12-13.

Straub et al., 2014 C. Straub, A.J. Granger, J.L. Saulnier, B.L. Sabatini. CRISPR/Cas9-mediated gene knock-down in post-mitotic neurons. PLoS One, 9 (2014), Article e105584.

Sun et al., 2011 F. Sun, K.K. Park, S. Belin, D. Wang, T. Lu, G. Chen, K. Zhang, C. Yeung, G. Feng, B.A. Yankner, Z. He. Sustained axon regeneration induced by co-deletion of PTEN and SOCS3. Nature, 480 (2011), pp. 372-375.

Swiech et al., 2015 L. Swiech, M. Heidenreich, A. Banerjee, N. Habib, Y. Li, J. Trombetta, M. Sur, F. Zhang. In vivo interrogation of gene function in the mammalian brain using CRISPR-Cas9. Nat. Biotechnol., 33 (2015), pp. 102-106.

Telese et al., 2015 F. Telese, Q. Ma, P.M. Perez, D. Notani, S. Oh, W. Li, D. Comoletti, K.A. Ohgi, H. Taylor, M.G. Rosenfeld. LRP8-Reelin-regulated neuronal enhancer signature underlying learning and memory formation. Neuron, 86 (2015), pp. 696-710.

Terns and Terns, 2011 M.P. Terns, R.M. Terns. CRISPR-based adaptive immune systems. Curr. Opin. Microbiol., 14 (2011), pp. 321-327.

Trotman et al., 2007 L.C. Trotman, X. Wang, A. Alimonti, Z. Chen, J. Teruya-Feldstein, H. Yang, N.P. Pavletich, B.S. Carver, C. Cordon-Cardo, H. Erdjument-Bromage, P. Tempst, S.G. Chi, H.J. Kim, T. Misteli, X. Jiang, P.P. Pandolfi. Ubiquitination regulates PTEN nuclear import and tumor suppression. Cell, 128 (2007), pp. 141-156.

Veres et al., 2014 A. Veres, B.S. Gosis, Q. Ding, R. Collins, A. Ragavendran, H. Brand, S. Erdin, C.A. Cowan, M.E. Talkowski, K. Musunuru. Low incidence of off-target mutations in individual CRISPR-Cas9 and TALEN targeted human stem cell clones detected by whole-genome sequencing. Cell Stem Cell, 15 (2014), pp. 27-30.

Wang et al., 2014 T. Wang, J.J. Wei, D.M. Sabatini, E.S. Lander. Genetic screens in human cells using the CRISPR-Cas9 system. Science, 343 (2014), pp. 80-84.

Wang et al., 2015 Z. Wang, A. Reynolds, A. Kirry, C. Nienhaus, M.G. Blackmore. Overexpression of Sox11 promotes corticospinal tract regeneration after spinal injury while interfering with functional recovery. J. Neurosci., 35 (2015), pp. 3139-3145. 
Welsbie et al., 2013 D.S. Welsbie, Z. Yang, Y. Ge, K.L. Mitchell, X. Zhou, S.E. Martin, C.A. Berlinicke, L. Hackler Jr., J. Fuller, J. Fu, L.H. Cao, B. Han, D. Auld, T. Xue, S. Hirai, L. Germain, C. SimardBisson, R. Blouin, J.V. Nguyen, C.H. Davis, R.A. Enke, S.L. Boye, S.L. Merbs, N. Marsh-Armstrong, W.W. Hauswirth, A. DiAntonio, R.W. Nickells, J. Inglese, J. Hanes, K.W. Yau, H.A. Quigley, D.J. Zack. Functional genomic screening identifies dual leucine zipper kinase as a key mediator of retinal ganglion cell death. Proc. Natl. Acad. Sci. U. S. A., 110 (2013), pp. 4045-4050.

Wu et al., 2014 X. Wu, A.J. Kriz, P.A. Sharp. Target specificity of the CRISPR-Cas9 system. Quant. Biol., 2 (2014), pp. 59-70.

Yang et al., 2013 H. Yang, H. Wang, C.S. Shivalila, A.W. Cheng, L. Shi, R. Jaenisch. One-step generation of mice carrying reporter and conditional alleles by CRISPR/Cas-mediated genome engineering. Cell, 154 (2013), pp. 1370-1379.

Yungher et al., 2015 B.J. Yungher, X. Luo, Y. Salgueiro, M.G. Blackmore, K.K. Park. Viral vector-based improvement of optic nerve regeneration: characterization of individual axons' growth patterns and synaptogenesis in a visual target. Gene Ther., 22 (2015), pp. 811-821.

Zou et al., 2009 H. Zou, C. Ho, K. Wong, M. Tessier-Lavigne. Axotomy-induced Smad1 activation promotes axonal growth in adult sensory neurons. J. Neurosci., 29 (2009), pp. 7116-7123.

Zou et al., 2015 Y. Zou, M. Stagi, X. Wang, K. Yigitkanli, C.S. Siegel, F. Nakatsu, W.B. Cafferty, S.M. Strittmatter. Gene-silencing screen for mammalian axon regeneration identifies Inpp5f (Sac2) as an endogenous suppressor of repair after spinal cord injury. J. Neurosci., 35 (2015), pp. 10429-10439.

Zuckermann et al., 2015 M. Zuckermann, V. Hovestadt, C.B. Knobbe-Thomsen, M. Zapatka, P.A. Northcott, K. Schramm, J. Belic, D.T. Jones, B. Tschida, B. Moriarity, D. Largaespada, M.F. Roussel, A. Korshunov, G. Reifenberger, S.M. Pfister, P. Lichter, D. Kawauchi, J. Gronych. Somatic CRISPR/Cas9-mediated tumour suppressor disruption enables versatile brain tumour modelling. Nat. Commun., 6 (2015), p. 7391.

Zukor et al., 2013 K. Zukor, S. Belin, C. Wang, N. Keelan, X. Wang, Z. He. Short hairpin RNA against PTEN enhances regenerative growth of corticospinal tract axons after spinal cord injury. $J$. Neurosci., 33 (2013), pp. 15350-15361. 